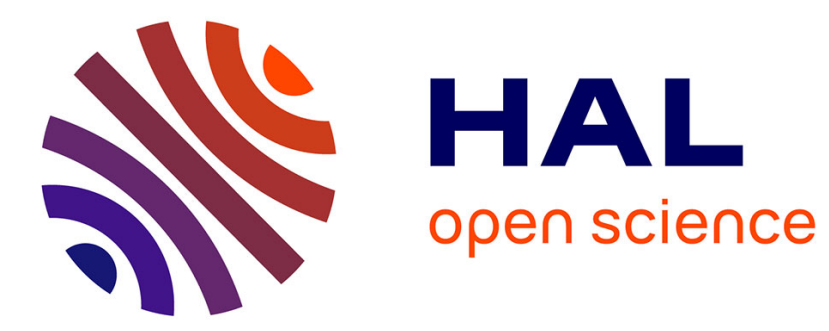

\title{
Une lecture démographique de la frontière Est-Ouest
}

Morgane Labbe

\section{To cite this version:}

Morgane Labbe. Une lecture démographique de la frontière Est-Ouest. Revue d'Etudes Comparatives Est-Ouest, 1995, 10.3406/receo.1995.2759 . hal-02984800

\section{HAL Id: hal-02984800 https://hal.science/hal-02984800}

Submitted on 1 Nov 2020

HAL is a multi-disciplinary open access archive for the deposit and dissemination of scientific research documents, whether they are published or not. The documents may come from teaching and research institutions in France or abroad, or from public or private research centers.
L'archive ouverte pluridisciplinaire HAL, est destinée au dépôt et à la diffusion de documents scientifiques de niveau recherche, publiés ou non, émanant des établissements d'enseignement et de recherche français ou étrangers, des laboratoires publics ou privés. 


\title{
Une lecture démographique de la frontière Est-Ouest
}

In: Revue d'études comparatives Est-Ouest. Volume 26, 1995, N4. pp. 55-74.

\begin{abstract}
A demographic interpretation of the East-West frontier.

Analysis of the effects of politics on populations helps towards an understanding of recent demographic developments in the countries of Eastern Europe. The greatest differences between Western Europe and Eastern Europe are to be found in their mortality rates. These differences arise from the public health systems, for the worsening of adult mortality was common to the whole of Europe. The higher mortality in East European countries can thus be explained by the problems of setting up a preventive policy, since this calls for the encouragement of a new kind of relationship between the individual and the institution. From this institutional perspective, it can be seen that the divide between East and West does not operate in family contexts, such as marriage, divorce or fertility, when the legal conditions were very different.
\end{abstract}

\section{Résumé}

L'analyse des effets du politique sur les populations est utile pour comprendre les évolutions démographiques récentes des pays de l'Europe orientale. C'est pour la mortalité que l'Europe de l'Ouest et l'Europe de l'Est présentent les plus grandes différences. Celles-ci relèvent des systèmes de santé car l'aggravation de la mortalité adulte fut commune à toute l'Europe. La surmortalité des pays de l'Est peut alors s'expliquer par les problèmes de mise en place d'une politique préventive, car celle-ci consiste à promouvoir un nouveau type de rapport entre l'individu et l'institution. Sous cet angle institutionnel, on peut alors comprendre que la coupure entre l'Est et l'Ouest ne s'observe pas pour des caractères familiaux tels que le mariage, le divorce ou la fécondité, alors que les législations étaient très différentes.

Citer ce document / Cite this document :

Labbé Morgane. Une lecture démographique de la frontière Est-Ouest. In: Revue d'études comparatives Est-Ouest. Volume 26, 1995, N4. pp. 55-74.

doi : $10.3406 /$ receo.1995.2759

http://www.persee.fr/web/revues/home/prescript/article/receo_0338-0599_1995_num_26_4_2759 


\title{
Une lecture démographique de la frontière Est-Ouest
}

\author{
Morgane LABBÉ*
}

A l'issue d'une séparation politique de près d'un demi-siècle, l'Europe de l'Est et l'Europe de l'Ouest présentent de grandes différences démographiques $^{1}$ : les niveaux de la mortalité et de la fécondité sont plus élevés, le mariage plus répandu et plus précoce. La disparition des régimes communistes soulève aujourd'hui la question de l'évolution démographique de ces pays : les différences se maintiendront-elles ? les convergences suivront-elles les proximités culturelles d'avant-guerre ? ou bien laisseront-elles la place à des modèles démographiques originaux dans lesquels l'expérience socialiste gardera une empreinte durable ? La question est d'autant plus importante que la situation démographique actuelle se caractérise par une baisse de la fécondité et une aggravation sanitaire ${ }^{2}$. Le délai est encore trop court pour qu'on puisse saisir dans les indices récents des changements démographiques profonds, mais la mesure de ces transformations conduit aussi à réapprécier l'effet du politique sur les populations.

Cette analyse s'impose pour deux raisons : si les indicateurs et les graphiques témoignent des divergences croissantes entre les deux parties de l'Europe, leurs géographies font aussi apparaître des disparités étrangères aux découpages politiques, mais présentant davantage de parentés avec des distributions plus anciennes. Cette diversité, insoupçonnée au regard des seuls indicateurs, étend la question des héritages de l'avant-guerre à la période actuelle. Mais ces géographies ne se répartissent pas suivant le modèle attendu : la mortalité, variable que l'on fait davantage dépendre du progrès économique que des politiques, donne la division la plus nette entre l'Est et l'Ouest tandis que

* Institut national d'études démographiques, Paris. Je remercie Alain Blum et Hervé Le Bras pour les recommandations qu'ils m'ont faites pour la rédaction de ce texte et tout au long des recherches qui l'ont précédé.

1. Pour une analyse détaillée de ces évolutions, voir le numéro de Population consacré aux pays de l'Est, $n^{\circ} 3,1989$.

2. Pour les indices les plus récents, voir A. Monnier et C. de Guibert-LANToine, «La conjoncture démographique », Population, $n^{\circ} 4-5,1994$. Une analyse des changements démographiques dans l'ex-RDA est faite par N. EBERSTADT, « Demographic Shocks After Communism : Eastern Germany 1989-93 », Population and Development Review, vol. $20, \mathrm{n}^{\circ} 1,1994$. 


\section{Morgane Labbé}

la fécondité, objet de politiques natalistes dans la plupart des régimes socialistes, s'avère moins sensible au découpage politique. Pour comprendre ces différences, on se départira de la notion de politique habituellement utilisée en démographie qui, restreinte à des secteurs d'activité de l'État - sanitaire ou familiale - conduit à rapporter les indicateurs au contenu des politiques et à mesurer leurs effets en termes d'efficacité. La notion de politique fait ici référence aux principes générateurs du social ${ }^{3}$. Cela revient à considérer les différents principes qui dictaient et légitimaient la mise en forme du social en Europe de l'Est et qui s'incarnaient dans des institutions et des normes, et à analyser en retour les pratiques, les réactions des populations - adaptation, rejet, référence à d'autres normes. C'est sous cet angle institutionnel et interactionniste ${ }^{4}$ que nous aborderons les différences démographiques européennes et cnvisagerons la question des héritages.

\section{La surmortalité de l'Europe de l'Est}

Au cours de l'après-guerre, le recul de la mortalité fut sensible dans tous les pays européens. C'est toutefois dans les pays de l'Europe orientale, devenue l'Europe de l'Est après la mise en place au lendemain de la guerre des régimes communistes, que les progrès ont été les plus importants. A partir de l'indicateur qui mesure la durée de vie moyenne - l'espérance de vie à la naissance -, on a calculé les gains enregistrés entre 1950 et 1988 pour chaque partie de l'Europe : ils s'élèvent, pour les hommes à 10 ans à l'Est au lieu de 7,4 ans à l'Ouest et, pour les femmes, respectivement à 12,8 ans et 9,8 ans. Ces progrès ont ainsi conduit au rattrapage des pays de l'Europe orientale sur ceux de l'Ouest, où la mortalité était plus faible au lendemain de la guerre : quatrecinq années séparent aujourd'hui leurs espérances de vie contre sept en 1950.

Cette comparaison rapide des indices actuels de la mortalité avec leurs valeurs observées quarante ans plus tôt montre une Europe de plus en plus homogène. Elle nous convaincrait de la résorption prochaine des derniers écarts entre l'Ouest et l'Est, ceux-ci témoignant du décalage chronologique qu'on observait déjà en 1950, et même avant-guerre, entre l'Europe orientale

3. On s'inspire ici de la philosophie politique et en particulier des travaux de Claude LEFORT, Esscais sur le politique, XIX ${ }^{e}-X X^{e}$ siècles, Seuil, 1986.

4. Sous l'angle de la micro-analyse, plusieurs études historiographiques ont ainsi rompu avec les deux approches qui prédominaient dans les sciences sociales, l'une situant les institutions à l'extérieur du social, l'autre au contraire le résumant. Elles ont mis en lumière les stratégies et les relations individuelles qui travaillent de l'intérieur les institutions, les consolident ou les modifient. Voir en particulier les recherches de Sabina LORIGA, Soldats. Un laboratoire disciplinaire : l'armée piémontaise au $X V I I I^{\prime}$ siècle, Mentha, 1991 ; et la synthèse de Jacques REVEL, "L'institution et le social », in Les formes de l'expérience, B. Lepetit (éd.), Albin Michel, 1985. 
et l'Europe occidentale. Mais, quand on retrace l'évolution de l'indicateur au cours de cette période, il s'avère que la majorité des progrès enregistrés en Europe de l'Est ont eu lieu au cours des dix-quinze premières années de l'après-guerre. Le déclin de la mortalité a été soudainement interrompu, le rattrapage de l'Ouest a en fait cessé dans les années soixante, il est resté depuis lors inachevé. Les écarts actuels entre les deux parties de l'Europe ne peuvent donc pas être expliqués par la persistance d'un décalage ancien : ils semblent davantage liés à la division politique entre l'Est et l'Ouest. Cette hypothèse nous conduit à étudier la géographie de la mortalité et à mettre en relation ses modifications au cours de cette période - l'émergence d'un clivage suivant la frontière Est-Ouest dans les années soixante-dix et son renforcement depuis lors - avec les évolutions de la mortalité.

\section{La géographie de l'espérance de vie à la naissance en Europe de 1950 à 1988}

Au lendemain de la guerre, l'Europe orientale se distinguait de l'Europe occidentale $^{5}$ par une mortalité plus élevée, mais cette division que fait apparaître la géographie de l'espérance de vie en 1950 (carte la) ne reflète pas encore leur récente séparation politique. Elle s'avère d'ailleurs très différente de celle qui surgira plus tard entre l'Est et l'Ouest, les pays de l'Europe orientale se situant toujours au même niveau que ceux de l'Europe méridionale, tout autant défavorisés sur le plan sanitaire. Rendre compte de cette géographie européenne selon des grands axes, Nord-Sud ou Ouest-Est, est en fait peu pertinent à cette date. Il existe plutôt une zone de basse mortalité, composée de quelques pays de l'Europe septentrionale (pays scandinaves, Grande-Bretagne, Pays-Bas, Suisse) et qui forment une sorte de pôle avancé du vieux continent, émergeant dans une Europe où les pays à mortalité élevée (avec une espérance de vie inférieure à 60 ans) constituent encore un territoire important. Cette zone s'étend non seulement sur toute l'Europe orientale et balkanique mais elle englobe aussi, à l'Ouest, des pays méditerranéens comme l'Espagne et le Portugal. C'est aussi à l'échelle de pays bordant la frontière Est-Ouest que les rapprochements empêchent de confondre les distributions de la mortalité des années cinquante et quatre-vingt : le niveau de la mortalité de la RDA est plus proche de celui de la RFA que de ceux des autres pays de l'Est, celui de l'Autriche est encore élevé en comparaison de celui de la Suisse ou de l'Allemagne fédérale et même de la RDA. Il est en fait plus proche de celui de la Hongrie ou de la Tchécoslovaquie, et révèle ainsi la permanence d'une Europe centrale que l'histoire et la culture réunissent encore au lendemain de la guerre en dépit des systèmes politiques différents qui les partagent déjà.

5. Dans cet article, les expressions Europe orientale-Europe occidentale renvoient à un découpage géographique aux frontières variables, Europe de l'Est-Europe de l'Ouest à la division politique de l'après-guerre. Nous avons inclus la Yougoslavie dans le groupe de l'Est. 
Morgane Labbé

Carte 1

La mortalité en Europe : durée de vie moyenne des hommes de 1950 à 1988

a) 1950
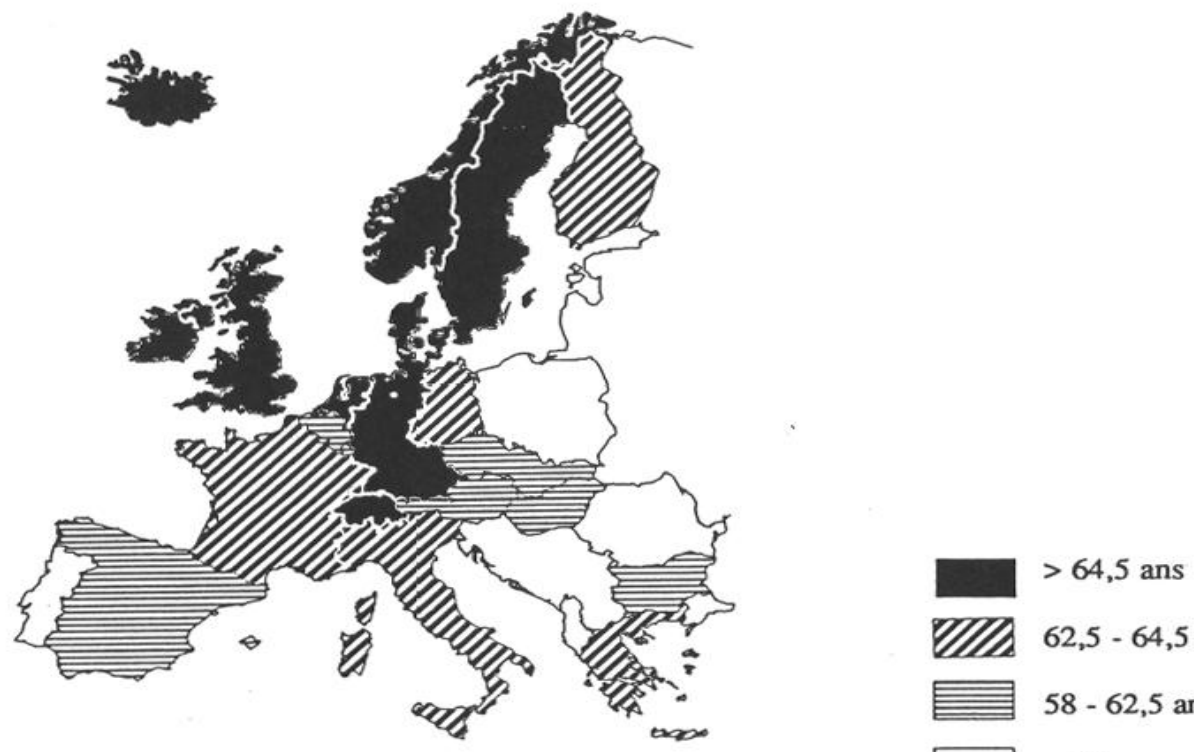

Q2, 62 - 64,5 ans

竖2 $58-62,5$ ans

$\square<58$ ans

b) 1960
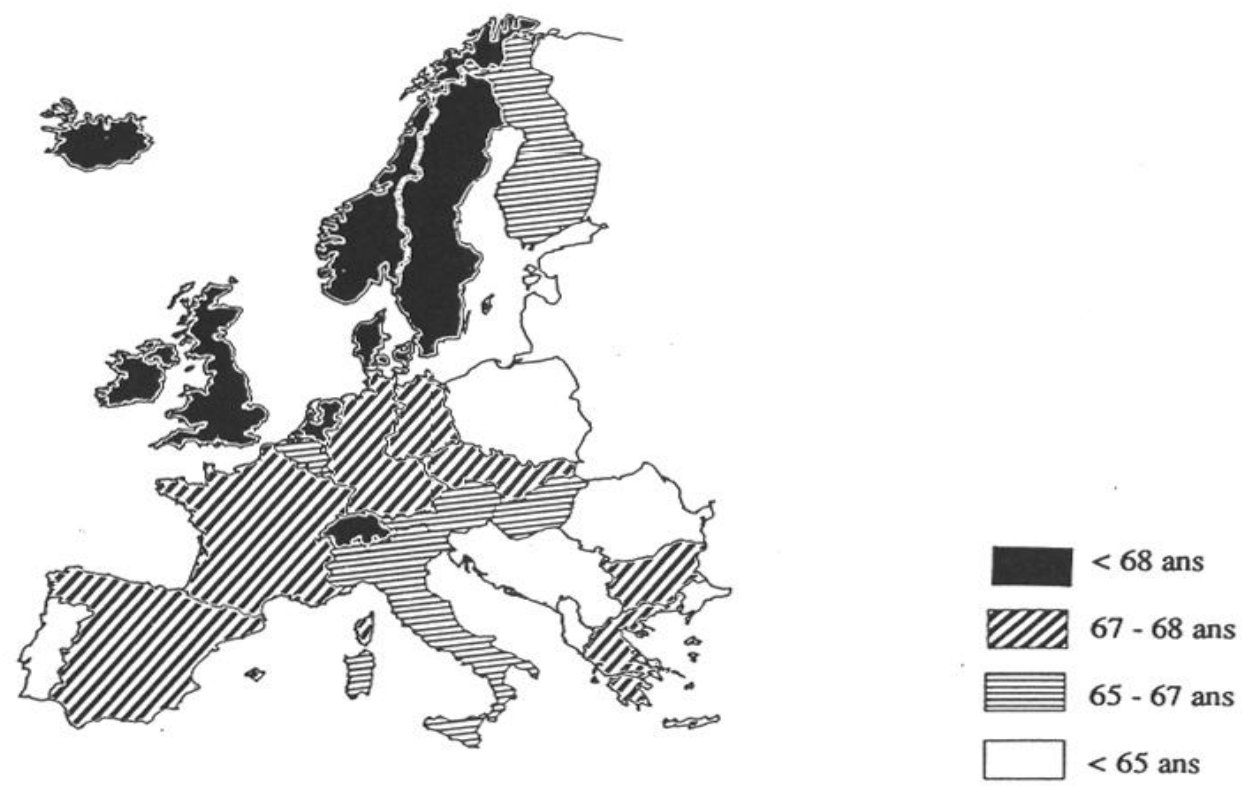
c) 1970

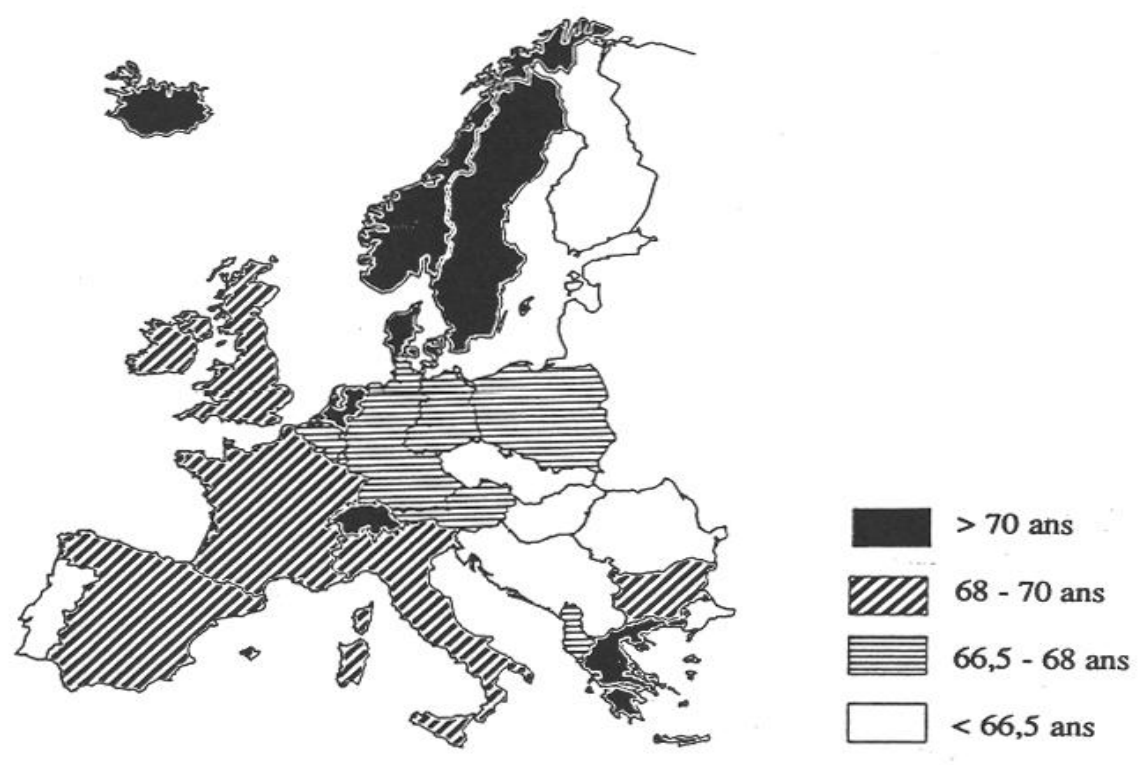

d) 1988

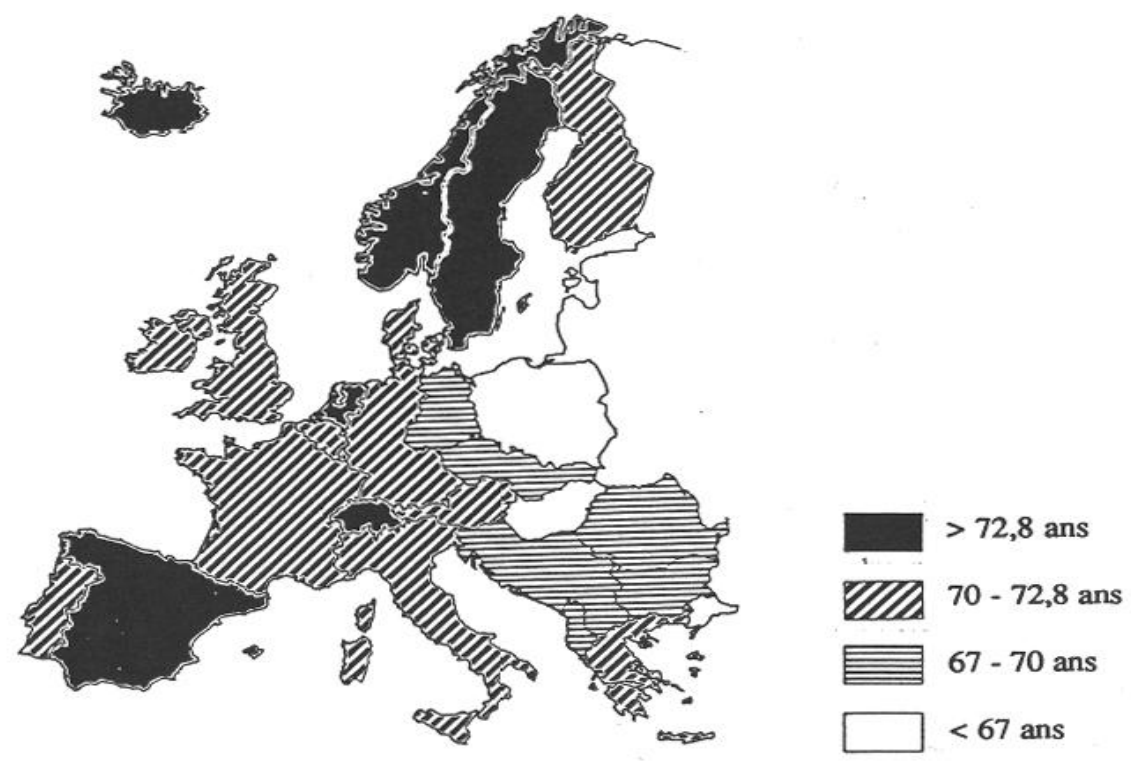


Dix ans plus tard, en 1960, cette géographie de la mortalité présente de grands changements : l'écart entre l'Europe centrale et l'Europe occidentale s'est considérablement réduit, mais il s'est accusé avec les pays du Sud (carte 1 b). Cette nouvelle division résulte de la chute rapide de la mortalité dans certains pays de l'Est, la Tchécoslovaquie ${ }^{6}$ notamment, mais l'écart avec les pays méditerranéens ne suit pas le découpage politique ; il s'accuse aussi entre pays danubiens et pays balkaniques. Cette géographie dure peu et, en 1970, les premiers signes d'un retournement apparaissent à l'Est (carte 1c). Les pays de l'Europe centrale, qui tendaient à se confondre avec ceux de l'Europe du Nord et de l'Ouest, cessent leur rapprochement et sont rattrapés par les pays balkaniques où se poursuit le recul de la mortalité, au départ plus élevée. Il n'y a pas de coupure entre l'Ouest et l'Est, mais seulement un mouvement d'unification des pays de l'Est. Le clivage nord-sud s'est fortement atténué, il a même disparu dans certains cas : l'Espagne, par exemple, où la mortalité n'a pas cessé de baisser, se trouve définitivement ancrée dans l'Europe de l'Ouest. Là, comme dans les autres pays du Sud, les écarts de mortalité résultaient surtout du décalage chronologique de la baisse, et la diffusion des progrès médicaux a permis de résorber le retard. Entre 1970 et 1980, la géographie de l'espérance de vie change peu. Les deux tendances à l'œuvre dès cette date - rattrapage du Sud, et stagnation des progrès à l'Est - se sont poursuivies, si bien que la coupure entre Europe de l'Ouest et Europe de l'Est s'est affirmée : la Grèce se sépare des autres pays balkaniques, la proximité de l'Autriche avec les autres pays danubiens de l'Est s'est également effacée. En 1988, la division est achevée, les exceptions ne sont plus politiques mais géographiques : la mortalité plus faible des pays comme la Grèce ou l'Autriche contraste avec celle de leurs pays voisins socialistes (carte 1d). Résumant l'ensemble de cette évolution, la carte de la répartition des gains d'espérance de vie enregistrés au cours des quatre décennies (carte 2) montre que le clivage actuel naît des mouvements de la mortalité de l'Europe de l'Est : la concentration des gains en début de période témoigne de la stagnation récente.

Les différences actuelles d'espérance de vie en Europe recoupent donc bien une division politique. Les composantes de cette surmortalité sont connues, c'est l'aggravation de la mortalité adulte qui est aujourdhui à l'origine des différences européennes. Mais il est utile de retracer la géographie pour saisir un fait moins souligné : les populations de l'Europe de l'Ouest furent exposées aux mêmes risques pathologiques et accusèrent pour la plupart une remontée des quotients de mortalité aux âges adultes ; cette hausse fut cependant provisoire alors qu'elle s'est maintenue à l'Est.

6. Cf. J. Rychtarikova, J. Vallin, F. Meslé, « Évolution comparée de la mortalité en République tchèque et en France depuis $1950 »$, Population, $\mathrm{n}^{\circ} 3,1988$. 


\section{Carte 2}

L'augmentation de la durée de vie des hommes en Europe : répartition des années gagnées entre 1950 et 1988

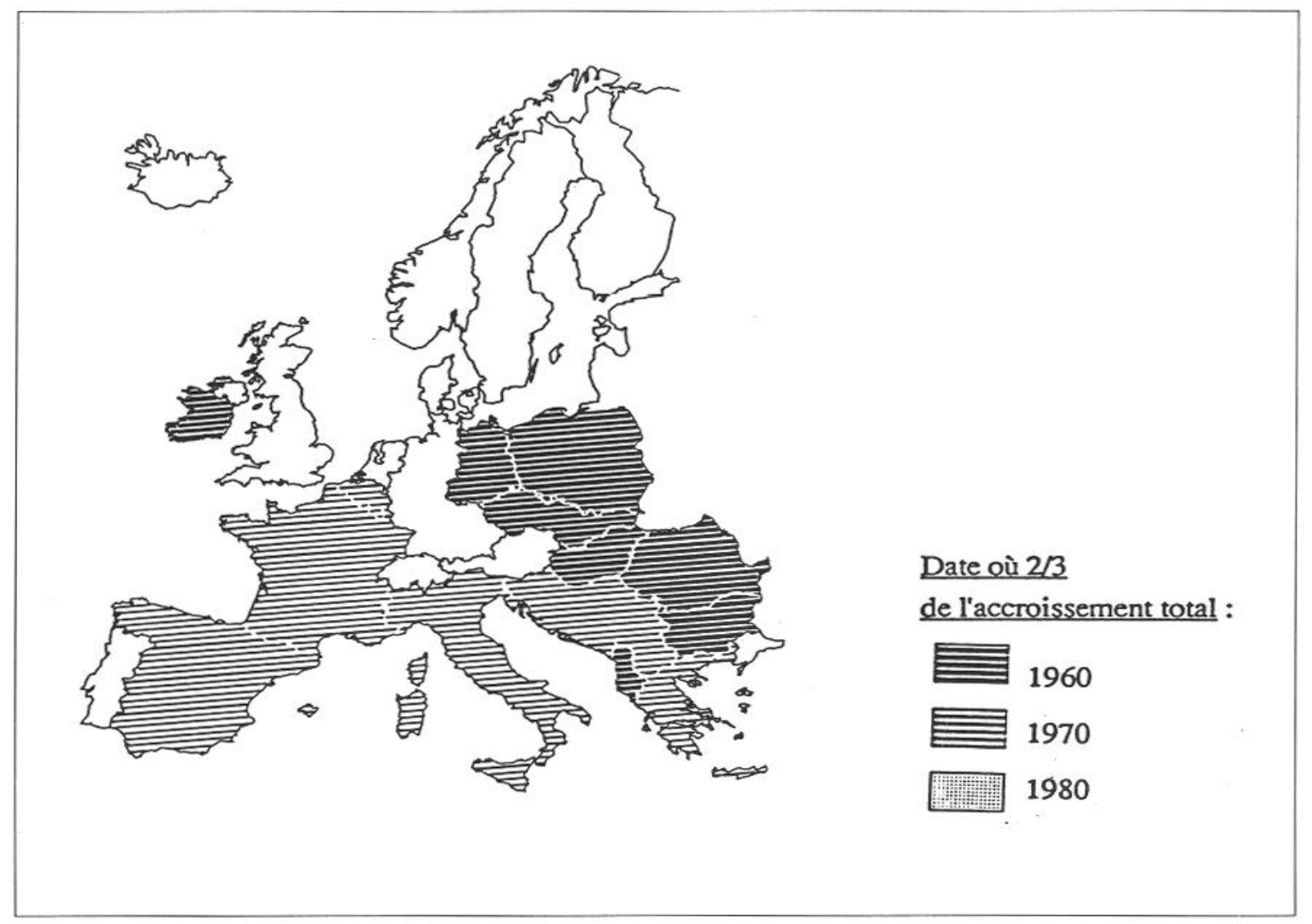

2. L'augmentation de la mortalité adulte

Depuis la dernière guerre mondiale, la baisse de la mortalité a été inégale selon l'âge. Dans les pays de l'Est comme dans toute l'Europe, la diminution de la mortalité causée par les maladies infectieuses a permis d'importants progrès aux jeunes âges?. Leur recul a commencé dans les années trente, grâce à la découverte et à la diffusion des sulfamides puis des antibiotiques, et il se poursuit dans les années de l'après-guerre. En conséquence, le poids de ces patho-

7. Voir le numéro spécial de Population, op. cit. Voir aussi Graziella CAselli, «L'évolution à long terme de la mortalité », in J.L. Rallu, A. Blum (éds.), Démographie européenne, vol. 2, INED, coll. « Congrès et colloques », 1991. 
logies dans la mortalité totale a, dans tous les pays européens, fortement régressé. Première cause de décès dans les années trente et responsables de 30 à $50 \%$ de la mortalité totale, elles en représentaient au début des années soixante entre 8 et $17 \%$. En 1985, leur part est devenue négligeable, elle varie entre 1,5 et $6 \%$. Aux âges suivants, les progrès ont été plus faibles, diminuant au fur et à mesure que l'âge s'élève pour devenir presque nuls aux âges adultes. Chez les hommes, en particulier entre 30 et 65 ans, la mortalité a même augmenté dans plusieurs pays. En Union soviétique, en Tchécoslovaquie et Hongrie, la hausse fut telle que l'espérance de vie enregistra une baisse, cas unique de renversement dans des pays à faible mortalité.

Pour comprendre cette évolution et saisir la spécificité de l'Europe de l'Est, il faut la replacer dans celle de toute l'Europe à la même époque. Conséquence du recul de la mortalité infectieuse, tous les pays - à des dates variables selon leur avancée, c'est-à-dire dès les années cinquante pour la Scandinavie et à partir des années soixante-dix pour l'Europe méridionale - sont entrés dans une phase de stagnation. Entre 1960 et 1970, dans plus de la moitié des pays situés à l'Est comme à l'Ouest, le risque de décès des hommes à 45-54 ans a augmenté ; dans presque tous les autres pays (France, GrandeBretagne par exemple), il est resté constant. Dans les rares pays (la Suisse, par exemple) où la mortalité adulte a continué de baisser, les progrès furent très faibles surtout si on les compare à ceux des décennies suivantes. Le ralentissement des progrès sanitaires s'explique par cette modification des causes de mortalité : à la place des pathologies infectieuses, les maladies de l'appareil circulatoire et les tumeurs forment les deux premières causes de mortalité. Leur part s'est accrue en proportion du recul des décès infectieux, mais elle a aussi augmenté pour des raisons liées aux modes de vie et à l'environnement propres aux sociétés industrielles.

Jusqu'en 1970, les niveaux de la mortalité des adultes étaient donc peu différents à l'Est et à l'Ouest ; les divergences apparaissent plus tard parce que la baisse de la mortalité reprend à l'Ouest seulement. Cette reprise s'avère même indépendante des niveaux de mortalité : la diminution est par exemple aussi forte au Portugal et aux Pays-Bas. A l'Est, en revanche, la mortalité continue à stagner, aucune reprise comparable à celle des pays de l'Ouest n'ayant été enregistrée jusqu'ici. C'est donc tardivement que l'Ouest et l'Est se différencient comme le montre la géographie de la mortalité adulte : les disparités s'alignent subitement dans les années quatre-vingt sur la frontière politique (carte $3 \mathrm{a}, \mathrm{b}, \mathrm{c}$ ). Leur distribution éclaire ainsi la géographie de la mortalité générale (espérance de vie). Mais, si elle permet de préciser les causes de l'aggravation sanitaire des pays de l'Est, l'analyse de la mortalité adulte montre aussi qu'elle ne leur fut pas spécifique. Ce qui leur est propre, c'est le maintien de cette surmortalité qui contraste avec la reprise de la baisse dans les pays occidentaux. 


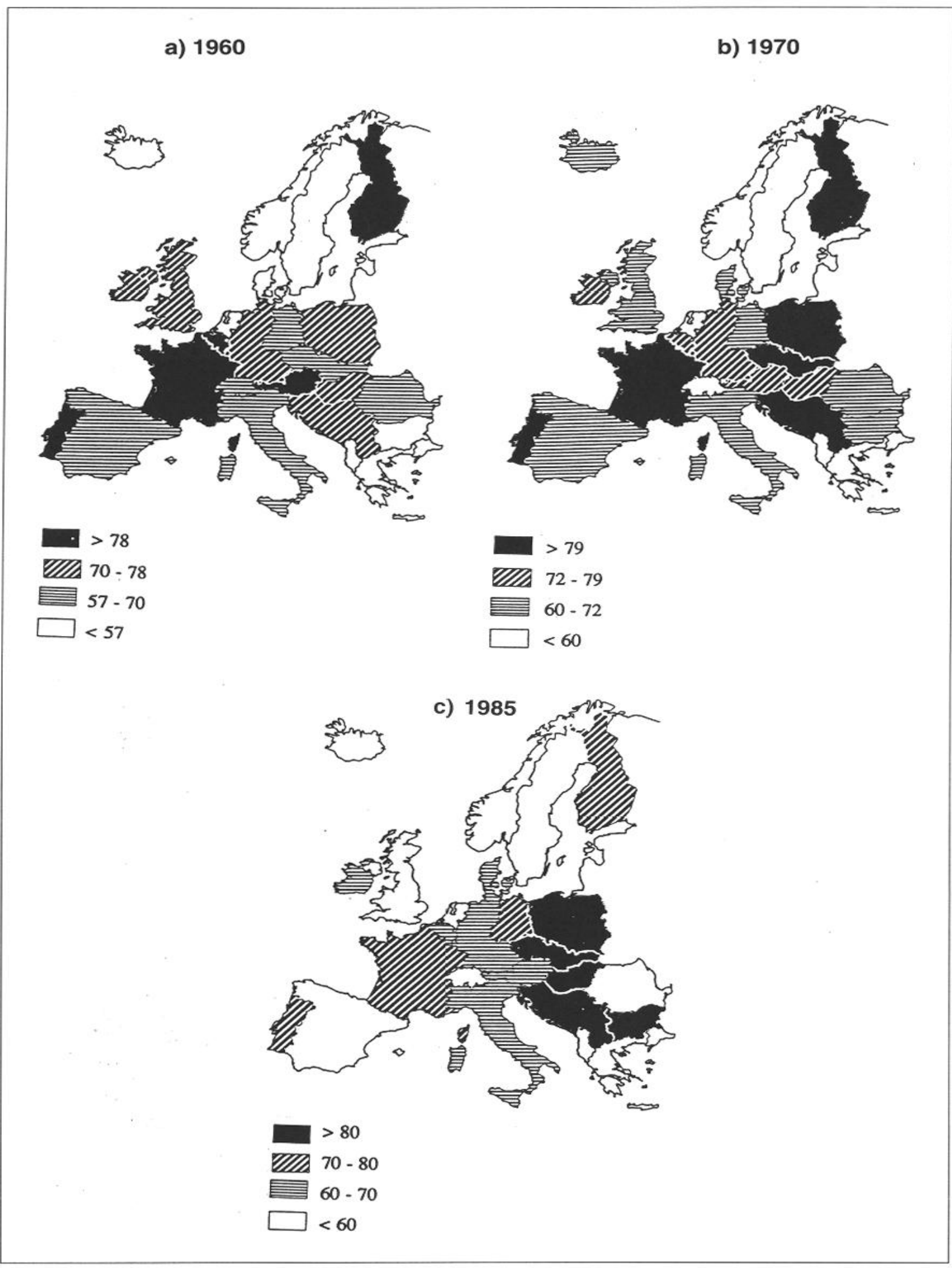




\section{Aggravation sanitaire et système de santé}

Forte et persistante comparée à celle qui affecta passagèrement les pays de l'Ouest, la hausse de la mortalité adulte des pays de l'Est suscita de nombreuses recherches. Comme dans les pays de l'Ouest, la plupart des maladies responsables des décès aux âges adultes ne résultent plus de l'action d'un facteur extérieur (viral ou infectieux), mais de déficiences physiologiques qui rendent les êtres plus vulnérables aux conditions d'existence individuelles. Les facteurs de risque associés à ces pathologies sont désormais connus pour tous les pays de l'Est. Ils sont essentiellement d'origine comportementale, liés aux habitudes de consommation excessive - de boissons alcoolisées, de cigarettes, etc. - ou à une alimentation déséquilibrée, trop riche ou au contraire carencée. Mais, une fois établie la liste de ces facteurs, peu d'explications ont ćtć proposées pour éclairer la situation inédite des pays de l'Est. Car les pays de l'Europe de l'Ouest ont été confrontés aux mêmes facteurs aggravants : les modes de vie ont comporté les mêmes excès ou insuffisances. On retrouve donc dans les pays de l'Est les principaux risques associés aux conditions de vie dans les sociétés industrielles. Ils ont été confrontés à des problèmes de vie matérielle de plus en plus semblables à ceux des pays de l'Ouest ${ }^{8}$. Aussi, l'augmentation dans les pays de l'Est des risques de mortalité aux âges adultes devrait-elle, en toute logique, être considérée comme le signe de leur rapprochement avec les pays de l'Ouest.

Comme dans ceux-là, l'augmentation des maladies cardio-vasculaires résulta d'abord de l'enrichissement des populations : un nombre croissant de gens eut accès à des produits jadis coûteux (cigarettes et alcools, beurre) ${ }^{9}$ dont ils ne songèrent pas à limiter la consommation. Mais les pays de l'Ouest ont pris rapidement en compte ces nouveaux déterminants et sont parvenus à enrayer la progression de ces pathologies en développant des techniques thérapeutiques adéquates et des programmes de prévention. Cette réussite se lit dans la baisse de la mortalité adulte qui a repris après dix-quinze années de stagnation. C'est donc la gravité des risques, leur persistance, et non leurs types, qui expliqueraient la surmortalité des pay's de l'Est. Cela conduit à déplacer le domaine de la recherche de celui des causes directes de la mortalité adulte à celui des systèmes de santé, puisque seuls les pays de l'Ouest sont parvenus à juguler l'expansion de cette mortalité.

8. Raymond Aron le prédisait dans les années soixante : «les manières de vivre tendront à se rapprocher des deux côtés du rideau de fer ; déjà elles tendent à se rapprocher. Il suffit d'une visite, même rapide, pour constater que beaucoup des soucis et considérations qui nous agitent en Occident se retrouvent sous une forme analogue (...), en fonction des mêmes données, la construction d'une société industrielle ». Démocratie et totalitarisme, Paris, Gallimard, 1965, coll. Folio/Essais, p. 329.

9. Quelques travaux font référence à cet enrichissement des populations pour expliquer l'aggravation de la mortalité aux âges adultes dans les pays de l'Est. Voir notamment, M. OKOLSKI sur la Pologne, dans La lutte contre la mort, J. Vallin et A. Lopez (éds.), INED-PUF, 1985, Travaux et Documents, 108, p.427, et G. CASELli, op. cit. 
C'est la faible réaction des systèmes de santé de l'Est qu'il faut en fait comprendre ${ }^{10}$. Celle-ci est rarement évoquée car éclipsée ou impliquée par des facteurs circonstanciels propres aux pays de l'Est - une forte récession économique et des problèmes de reconversion de leurs systèmes de santé pour répondre aux nouvelles pathologies. Il est vrai que leur influence ne peut pas être négligée. La récession économique des pays de l'Est a en effet conduit à une détérioration des conditions d'existence, tant au niveau individuel que collectif. Celle-ci rejaillit sur toute la qualité de la vie et a pu aussi ralentir, voire compromettre, la mise en place des structures sanitaires exigées par les nouvelles pathologies. Qu'il s'agisse de la création de nouveaux services, de l'acquisition de matériel, de la formation et du recrutement du personnel, tous avaient nécessairement un coût. Pourtant, le rôle prépondérant joué par la prévention dans la lutte contre les nouvelles pathologies rend secondaires ces aspects économiques : la lutte contre les pathologies cardio-vasculaires dépend moins des infrastructures que des comportements. Mais évaluer l'impact des politiques sanitaires sur les comportements conduit à doubler l'analyse démographique d'une analyse sociologique, qui s'intéresse aux relations entre la population et le système de santé.

\section{Les systèmes de santé des pays de l'Est face à la prévention individuelle}

L'efficacité des programmes occidentaux est due à la mise en place d'une nouvelle organisation médicale, distincte de celle requise pour lutter contre les maladies d'origine infectieuse. Elle associe aux interventions curatives sur l'organisme des actions dissuasives sur les comportements. Celles-ci attribuent un rôle croissant et privilégié aux programmes préventifs. Mais élargir le domaine de l'action sanitaire aux comportements individuels suppose un autre type de rapport entre les individus et les institutions sanitaires. Cet aspect relationnel a été négligé, car les recherches se sont d'abord préoccupées des défaillances internes aux organisations planifiées. L'inadaptation du système de santé des pays de l'Est aux nouvelles pathologies adultes a été considérée comme une cause majeure de leur retard sur l'Europe de l'Ouest. Mais les performances réalisées par ces systèmes laissaient penser qu'à terme ils seraient parvenus à infléchir la mortalité et à rejoindre le niveau des pays de l'Ouest. Le recul spectaculaire des maladies infectieuses dans les années cinquantesoixante attestait d'une efficacité à laquelle on pût encore se fier pour lutter contre les nouvelles pathologies. L'organisation planifiée a pu être aussi plus adaptée à la lutte contre les maladies d'origine infectieuse, celles-ci représentant une cause majeure de la mortalité lors de sa mise en place. L'organisation de la santé s'est donc trouvée marquée par les orientations sanitaires

10. Nous suivons ici Alain Blum qui, pour expliquer l'aggravation de la mortalité en U.R.S.S., s'est intéressé au rapport entre le système de santé et la population. Cf. A. Blum, Naître, vivre et mourir en L.R.S.S., 1917-1991, Plon, 1994. 
anciennes, celles-ci étant parfois antérieures à l'introduction des systèmes planifiés". Face aux nouvelles pathologies, les avantages de l'époque se sont alors mués en inconvénients. Les performances d'autrefois ont peut-être eu aussi un effet dissuasif sur la reconversion du système de santé exigée par les nouvelles pathologies. Conséquence de cette politique sanitaire, les pays de l'Est ont été confrontés au grave problème de la reconversion de leurs structures médicales ${ }^{12}$. Réaction tardive et reconversion difficile seraient responsables du retard des pays de l'Est. C'est un décalage que refléteraient les différences de mortalité entre l'Est et l'Ouest. Cette explication suppose qu'une fois leur reconversion achevée, les systèmes planifiés seraient à leur tour parvenus à faire baisser la mortalité d'origine cardio-vasculaire. Mais il se peut aussi que les conditions de réussite de cette reconversion n'aient pas été satisfaites. Nous développerons cette hypothèse en montrant que les principes de la prévention individuelle ne pouvaient pas être pris en compte par les régimes politiques des pays de l'Est.

\section{Les principes de la prévention individuelle}

Les politiques préventives détiennent un rôle primordial dans la lutte contre les maladies cardio-vasculaires, puisqu'elles permettent de modifier les habitudes de consommation et les comportements qui augmentent leurs prévalences. Pour les appliquer et les rendre effectives, les autorités disposent d'un large éventail de moyens, variant entre l'incitation et la cœercition. Tous les régimes politiques ont recours à des mesures contraignantes pour l'individu, qui interdisent, ou pour le moins tendent à limiter certains comportements. Ce qui justifie qu'ils soumettent les individus à des restrictions - et les pénalisent quand ils ne les respectent pas - puisque c'est la vie d'autrui qu'ils mettent en péril. Même des obligations médicales qui visent en apparence la santé individuelle, comme les vaccinations ou les consultations prénatales, prennent en compte les effets sur autrui, tels que les risques de contamination ou la santé du futur nouveau-né. Mais dès qu'il s'agit de modifier des attitudes qui ne menacent que la santé de chacun, les pouvoirs publics ne disposent pas de la même légitimité pour agir sur l'individu : la question est de savoir qui, en dernier ressort, définit ce qui est bien pour l'individu. C'est là que divergent les régimes politiques : à la différence des pays de l'Ouest, les pays de l'Est ont

11. Si le modèle soviétique a été adopté au lendemain de la guerre par les pays de l'Est, celui-ci s'est probablement appuyé et inspiré de l'organisation sanitaire de l'époque tsariste. Cf. A. Blum, Naître..., op. cit.10. Nous suivons ici Alain Blum qui, pour expliquer l'aggravation de la mortalité en U.R.S.S., s'est intéressé au rapport entre le système de santé et la population. Cf. A. BLUM, Naître, vivre et mourir en U.R.S.S., 1917-1991, Plon, 1994.

12. Voir, par exemple, Eva Orosz, «La santé publique en Hongrie. L’héritage du socialisme et la réforme », Revue française des Affaires sociales, $\mathrm{n}^{\circ}$ 1, 1992. 
adopté des programmes beaucoup plus restrictifs pour modifier les comportements ${ }^{13}$. Qu'ils aient été délibérément préférés à des mesures plus incitatives, ou destinés à renforcer celles-ci jugées inefficaces, une question reste : une politique de prévention moins restrictive aurait-elle réussi comme dans les pays de l'Ouest à faire reculer la mortalité des adultes ?

La faiblesse des programmes de prévention des pays de l'Est est souvent présentée comme une défaillance majeure de leur politique de santé, expliquant ainsi les écarts de mortalité avec l'Ouest. Mais il se peut aussi que ce retard ou les difficultés d'application des programmes préventifs résultent de contradictions existant entre les principes de la prévention individuelle et ceux sur lesquels se fonde l'ensemble de leur système politique et social. En effet, une politique préventive non restrictive suppose la liberté du choix individuel et le principe de la responsabilité qui lui est associée. Elle reconnaît la vie privée, domaine de l'existence dans lequel l'individu agit en fonction d'autres normes, qu'elles soient familiales, religieuses ou autres. Dans les régimes démocratiques, la prévention doit accepter cette autonomie individuelle même si celle-ci contrecarre ses objectifs et limite son efficacité. Elle admet la diversité des choix et des comportements, mais surtout elle doit intégrer dans son action le principe d'un choix, décidé en fonction d'intérêts particuliers : pour que l'individu entreprenne de modifier ses habitudes, il faut qu'il dispose en permanence de la liberté de s'y soumettre ou au contraire de s'en affranchir. Lui seul peut s'y astreindre, car alors il l'entreprend dans son intérêt propre, intérêt qui lui appartient et qu'il modifie à son gré ${ }^{14}$. Aussi, l'une des voies la plus souvent empruntée par les programmes de prévention est-elle celle des médias diffusant dans toute la population les informations sur les risques ou les gains associés à tel ou tel comportement. Tout individu, supposé averti, est censé agir par la suite de manière rationnelle. Mais cette rationalité reste individuelle, c'est-à-dire qu'elle relève d'intérêts multiples et privés, et donc inconnus. Elle signifie seulement que l'individu a opéré un choix en vertu de cette connaissance et de sa liberté de comportement. Dans la réalité, la circulation de l'information est imparfaite et la liberté dont jouit chaque personne est relative, mais ces garanties institutionnelles restent néanmoins indispensables à la réalisation des programmes de prévention individuelle. Ces principes sont politiques, ils fondent les seules démocraties libérales.

13. Comme, par exemple, la politique de lutte contre l'alcoolisme adoptée par le régime soviétique dans les années quatre-vingt, qui consistait à réduire considérablement la production et la consommation d'alcool et à en sanctionner sévèrement la consommation.

14. Dans le débat en France divisant le corps médical sur la question du dépistage prénatal obligatoire du SIDA, ceux qui y étaient opposés développèrent le même raisonnement, liant prévention et liberté individuelle. Ils évoquèrent l'efficacité limitée d'une telle mesure, avec les risques d'accouchements clandestins de femmes refusant de se soumettre au dépistage. 


\section{L'hypothèse d'un rejet des services de santé}

Le lien entre système politique et niveau de santé peut être étudié non plus à travers les structures médicales, mais du point de vue de la population ${ }^{15}$. On peut en effet concevoir une désaffection provenant des individus, qui se détourneraient volontairement des services de santé ou se désintéresseraient de leurs recommandations. Ce rejet des services de santé serait associé à un rejet plus général de tout service de l'État, moins en raison du caractère restrictif de certaines mesures, ou simplement de leur inefficacité avérée, que comme conséquence d'une défiance croissante envers l'administration. Certes, tous les systèmes de santé, à l'Est comme à l'Ouest, ont recours à des services administratifs aux tendances bureaucratiques. Les individus se trouvent là dans un rapport analogue à celui qu'engendre n'importe quelle autre administration de l'État. Mais dans les systèmes de santé occidentaux, ce rapport tutélaire et de contrôle se trouve pondéré par un rapport contractuel, qui transforme ou dédouble le patient en client et l'autorise à rompre les liens avec le médecin et les services.

Envers tout service de l'État, il y aurait aussi des attitudes faites d'apathie et de négligence ${ }^{16}$ ou au contraire de résistance. La suspicion et la méfiance qu'inspirent les services médicaux sont des réactions générales à toutes les sociétés développées, où les prestations et les modes de protection collectifs nécessitent le recours à des services administratifs. Mais à un degré plus prononcé que dans les autres institutions publiques, ils enserrent l'individu, ils pénètrent très loin dans sa vie privée, étant en mesure de s'en approprier les détails. La santé vient toujours légitimer cette emprise sur l'individu : elle justifie sur le plan administratif les enregistrements d'informations diverses sur la vie du patient, elle rend anodine les contrôles individuels au moyen de la surveillance médicale et de la fréquence des consultations. Les exigences médicales, qui reculent ainsi la limite entre public et privé, peuvent converger avec des volontés politiques qui ne reconnaissent pas le principe d'une sphère privée dans l'existence individuelle. Ce rapport étroit et régulier entre l'individu et l'institution médicale facilite l'intrusion du politique dans la vie individuelle. Fait paradoxal, il apparaît plus privilégié que dans le domaine de la fécondité, objet de politiques spécifiques. Mais le contact institutionnel y est

15. Sur cette hypothèse et son développement, cf. Morgane LABBÉ, Dynamique politique des populations à différentes échelles européennes, Thèse EHESS (nouveau régime), 1995.

16. C'est'la situation de la Pologne où, selon M. Okolski, « les programmes nationaux (protection maternelle et infantile, prévention et traitement de l'alcoolisme, lutte contre la tuberculose, élimination des maladies vénériennes) n'ont qu'un piètre succès. En 1980, par exemple, la moitié des accouchées avaient consulté un spécialiste au cours du premier trimestre de leur grossesse »; cf. « Le cas de la Pologne », in La lutte contre la mort, op. cit. 
plus lâche, ponctuel, voire inexistant. Le contrôle de la reproduction par les familles se dérobe encore à l'intervention publique ${ }^{17}$.

\section{Efficacité et légitimité : le politique à l'épreuve des convergences}

L'explication politique ne se justifie pas seulement parce que la géographie des populations et celle des systèmes politiques se sont trouvé coïncider au cours de la période récente. Il s'agit, dans le sens des propos de Claude Lefort, de « faire retour à la question qui guidait autrefois la philosophie politique : qu'en est-il de la différence des formes de sociétés ? ${ }^{18}$. Cette préoccupation anthropologique est celle qui guide notre analyse des pays de 1'Est. Cela ne revient pas à traiter tous les faits sociaux comme politiques, mais à considérer les différents principes qui dictent la mise en forme du social dans un régime démocratique et dans un régime communiste, et qui s'incarnent dans les institutions, les règlements et les normes organisant la vie collective. Cependant, la société n'est pas résumée par ces principes. Distinguer principes et faits demeure une condition de la compréhension du social : aux normes imposées, les individus et les groupes réagissent, ils sont aussi producteurs de normes et se réfèrent à d'autres systèmes normatifs. Mais le fait que la pluralité des normes soit légitimée, ou non, relève du politique et a des implications sur le social.

La prise en compte du politique ne peut être confinée aux faits où les différences entre l'Est et l'Ouest sont les plus manifestes, elle doit s'étendre à l'ensemble de l'analyse sociale. La démographie doit ainsi interroger les ressemblances formelles et se garder de l'illusion de la convergence. Elle ne peut ignorer le politique quand les évolutions convergent et le solliciter quand elles divergent. Ainsi, jusque dans les années soixante-dix, le rapprochement des niveaux de mortalité entre l'Europe de l'Est et l'Europe de l'Ouest invite à évincer le politique. Pourtant s'il témoigne des performances des systèmes de santé socialistes et des qualités de leur protection sociale ${ }^{19}$, il s'opère là dans un ensemble politique distinct : l'efficacité des systèmes sanitaires ne doit pas rendre superflue la question de la légitimité. Ce serait sinon considérer les sociétés européennes comme des entités apolitiques qui auraient adopté des modes alternatifs de développement sanitaire, ceux-ci n'étant conçus que comme des systèmes techniques à leur disposition. Il ne s'agit pas alors d'introduire un jugement sur la légitimité des régimes, mais de prendre en compte le fait qu'ils agissent en fonction de définitions différentes de ce qui est légitime.

17. Alain Blum conclut aussi à une indépendance plus grande des comportements familiaux. Cf. Naître..., op. cit.

18. C. LEFORT, « La question de la démocratie », in Essais sur le politique..., op. cit.

19. Voir le bilan de Michel VoIRIN, «La sécurité sociale dans les pays d'Europe centrale et orientale : réformes et continuité », Revue internationale de sécurité sociale, vol. $46, \mathrm{n}^{\circ} 1,1993$. 


\section{L'Europe de l'Est face aux familles}

Les courbes de fécondité des pays de l'Est, par contraste avec celles des pays de l'Ouest, suggèrent à première vue une sensibilité plus grande de la fécondité au politique : les fluctuations nombreuses retracent les changements législatifs ${ }^{20}$ - libéralisation et restriction de l'avortement notamment - mais aussi l'adoption dans les années soixante-dix de mesures natalistes avantageuses, destinées à relever le niveau de la fécondité. Face aux mesures restrictives, comme l'interdiction de l'avortement, les populations réagissent toujours : l'augmentation du nombre de naissances est soudaine et forte durant quelques années. Mais il s'agit seulement d'une réaction à une contrainte imprévue, les comportements s'adaptant ensuite. C'est le même type de réaction qu'on observe aujourd'hui dans les anciens pays de l'Est, qui ont, à des degrés divers, abandonné ou révisé les législations familiales antérieures. De même, l'introduction d'une politique familiale distribuant de nouvelles prestations, réservant des avantages aux familles, donnant des garanties aux mères actives, peut conduire les couples à avancer la venue d'un enfant. Le nombre d'enfants augmente ainsi pendant quelques années. Comme presque tous les pays de l'Est ont adopté des politiques natalistes, on s'attendrait à trouver, comme pour la mortalité, une unification croissante de cette partie de l'Europe et une coupure aussi marquée aujourd'hui avec l'Ouest. La division devrait être d'autant plus nette que, selon les indices de fécondité par génération (descendance finale) récemment disponibles, ces politiques familiales auraient conduit à l'augmentation des naissances de deuxième rang ${ }^{21}$. Cependant, la géographie de la fécondité de la fin des années quatre-vingt ne restitue pas la division politique entre l'Est et l'Ouest (carte 4). Pour expliquer cette géographie, il faut rattacher les évolutions des dernières décennies aux tendances d'avant-guerre et prendre en compte l'influence de caractéristiques telles que la religion ou les structures familiales.

La différence avec la mortalité est donc frappante. Comment expliquer alors que le politique ait autant marqué les comportements sanitaires et si peu la fécondité. La différence pourrait d'abord s'expliquer par les moyens institutionnels qui permettent dans chaque cas la mise en forme du social par le politique : les institutions de santé n'ont pas leur équivalent pour la famille. Mais surtout, dans le cas de la famille comme dans celui de la prévention sanitaire, l'action de l'État n'est pas seulement prestataire ou distributive, elle comporte aussi une dimension normative face à laquelle les individus ou les groupes peuvent réagir en sollicitant d'autres normes ou valeurs puisées dans le passé. C'est cette dynamique des comportements familiaux que révèle la variété des

20. C'est ainsi que procède Patrick FESTY, « La fécondité en Europe de l'Est », Population, $\mathrm{n}^{\circ} 3,1991$.

21. Ibid. 


\section{Carte 4}

La fécondité en Europe en 1989 (Indicateur conjoncturel de fécondité)

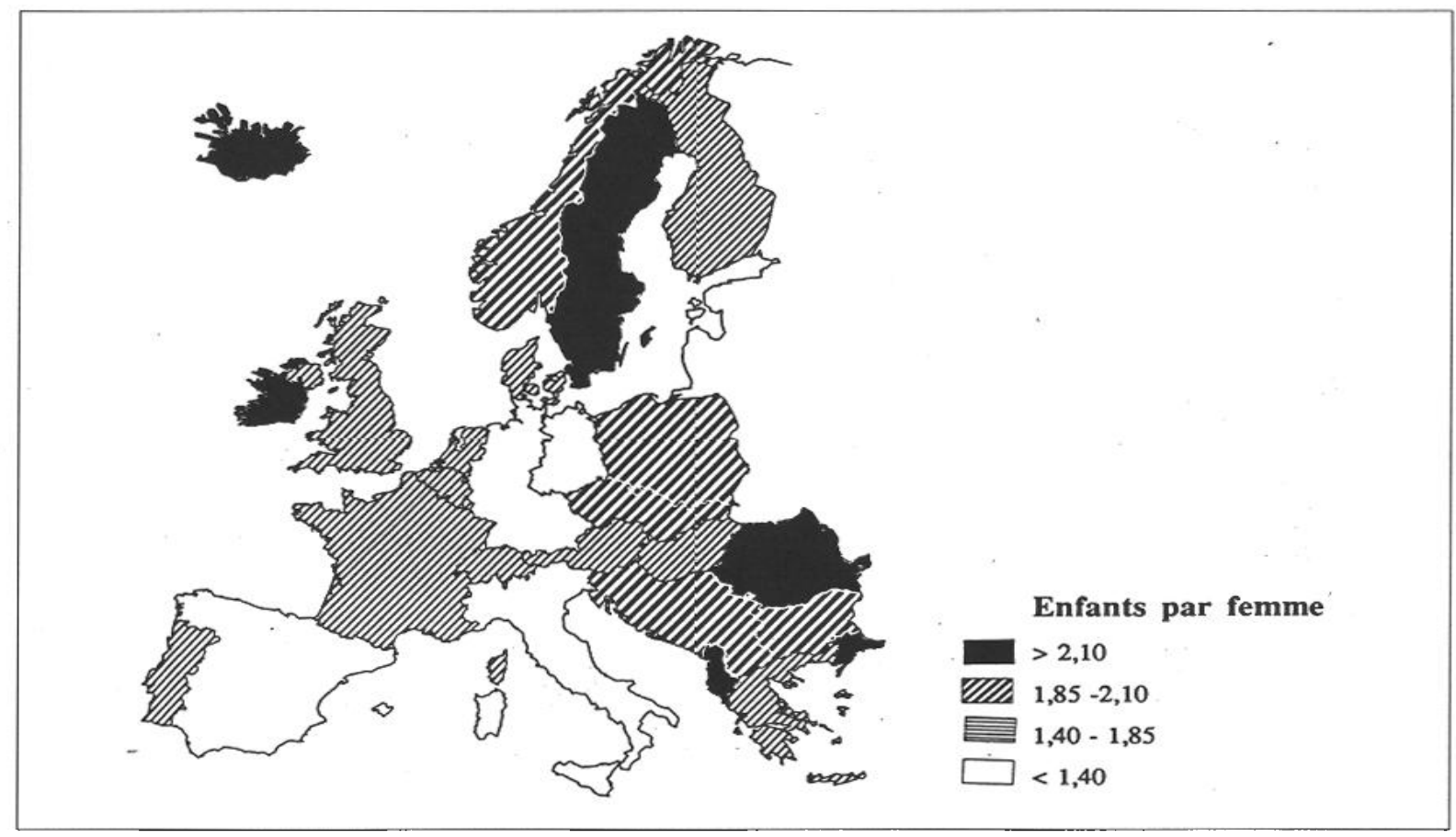

distributions démographiques et leur apparente incohérence. Pour les âges au mariage, ou ceux des femmes à la naissance de leur premier enfant, les différences européennes suivent très nettement la division Est-Ouest (carte 5a). Cette unité de l'Est résulte en fait de comportements adaptatifs : les âges sont précoces parce que l'accès au logement et à l'emploi sont facilités et que les familles sont avantagées par rapport aux célibataires. Cette souplesse des calendriers familiaux a d'ailleurs été observée dans de nombreuses situations historiques ${ }^{22}$; l'âge comporte pour les populations européennes un enjeu essentiellement économique, l'enjeu symbolique et normatif étant réduit. Il se fixe sur d'autres attitudes familiales comme le montre l'absence d'unité de l'Europe de l'Est en ce qui concerne la taille des familles, la part des naissances hors mariage et la fréquence du divorce : ces caractéristiques forment des géographies très différentes (cartes $4,5 \mathrm{~b}, 6,7$ ). Même si les politiques natalistes favorisent la venue du troisième enfant, le nombre d'enfants relève d'autres rationalités, qu'elles soient religieuses, familiales ou autres. La géographie du divorce, comme celle de l'illégitimité, font ainsi ressortir des clivages plus anciens entre pays, voire entre régions.

22. Cette sensibilité du mariage a été bien mesurée dans le cas de l'Angleterre préindustrielle par E.A.Wrigley et R.S. SCHOFIEld, The Population History of England, 1541-1871 : a Reconstruction, Harvard University Press, 1981. Voir également, Hervé Le Bras, Les Trois France, Paris, Odile Jacob, (1986), nouvelle édition, 1995. 


\section{Carte 5 \\ Le mariage en Europe en 1988}

a) L'âge au premier mariage des femmes

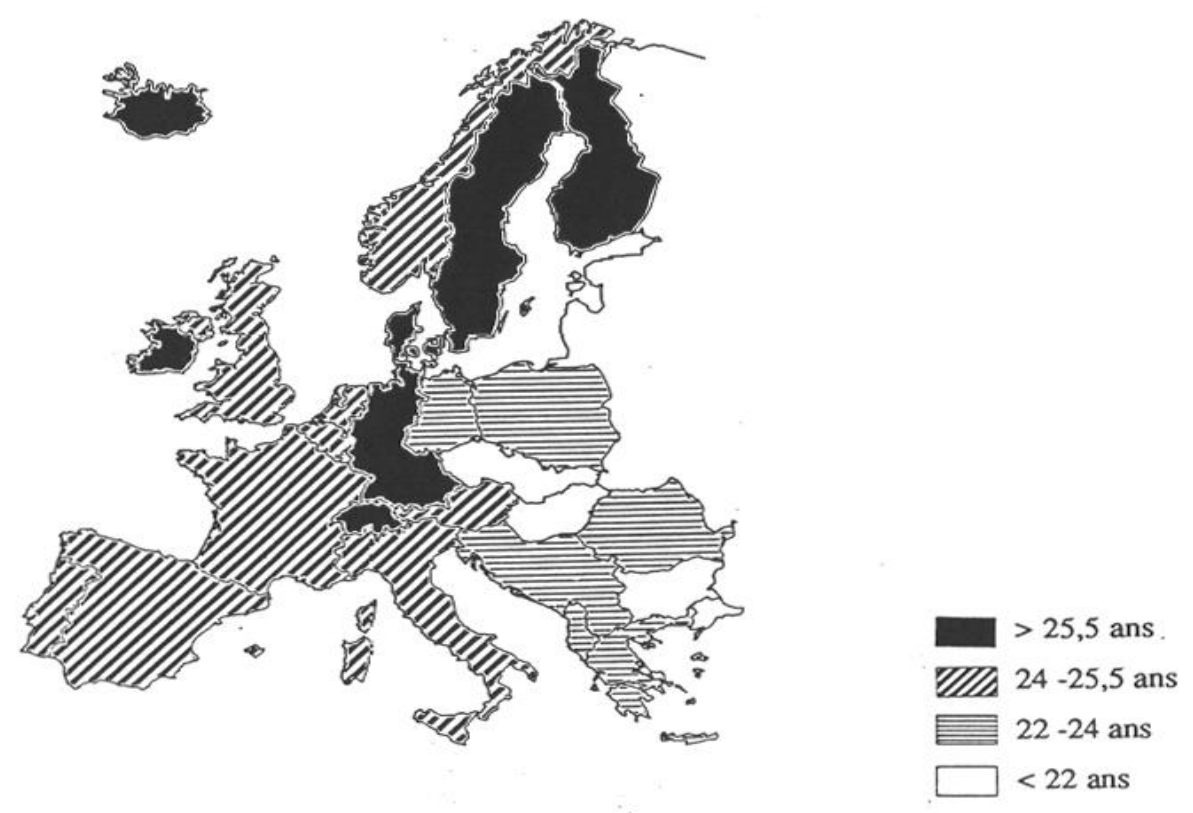

b) Écarts d'âge au mariage entre hommes et femmes
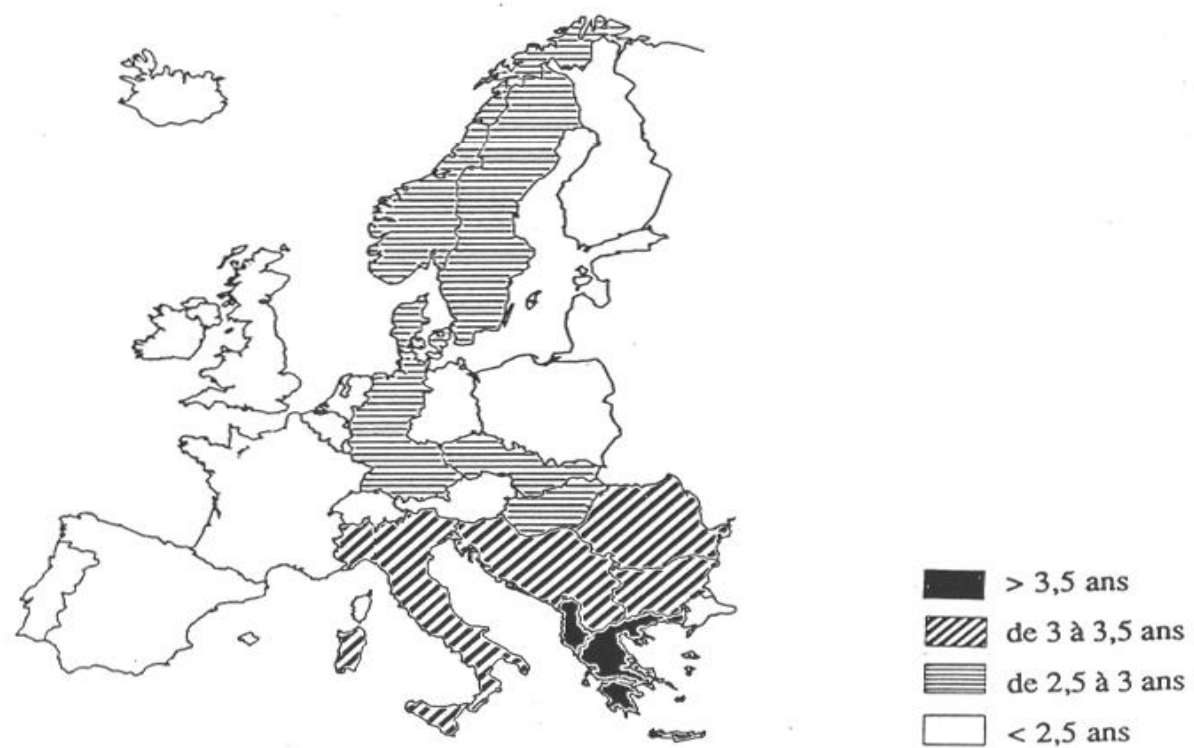
Carte 6

Le divorce en Europe en 1988 (Indice conjoncturel de divorcialité)

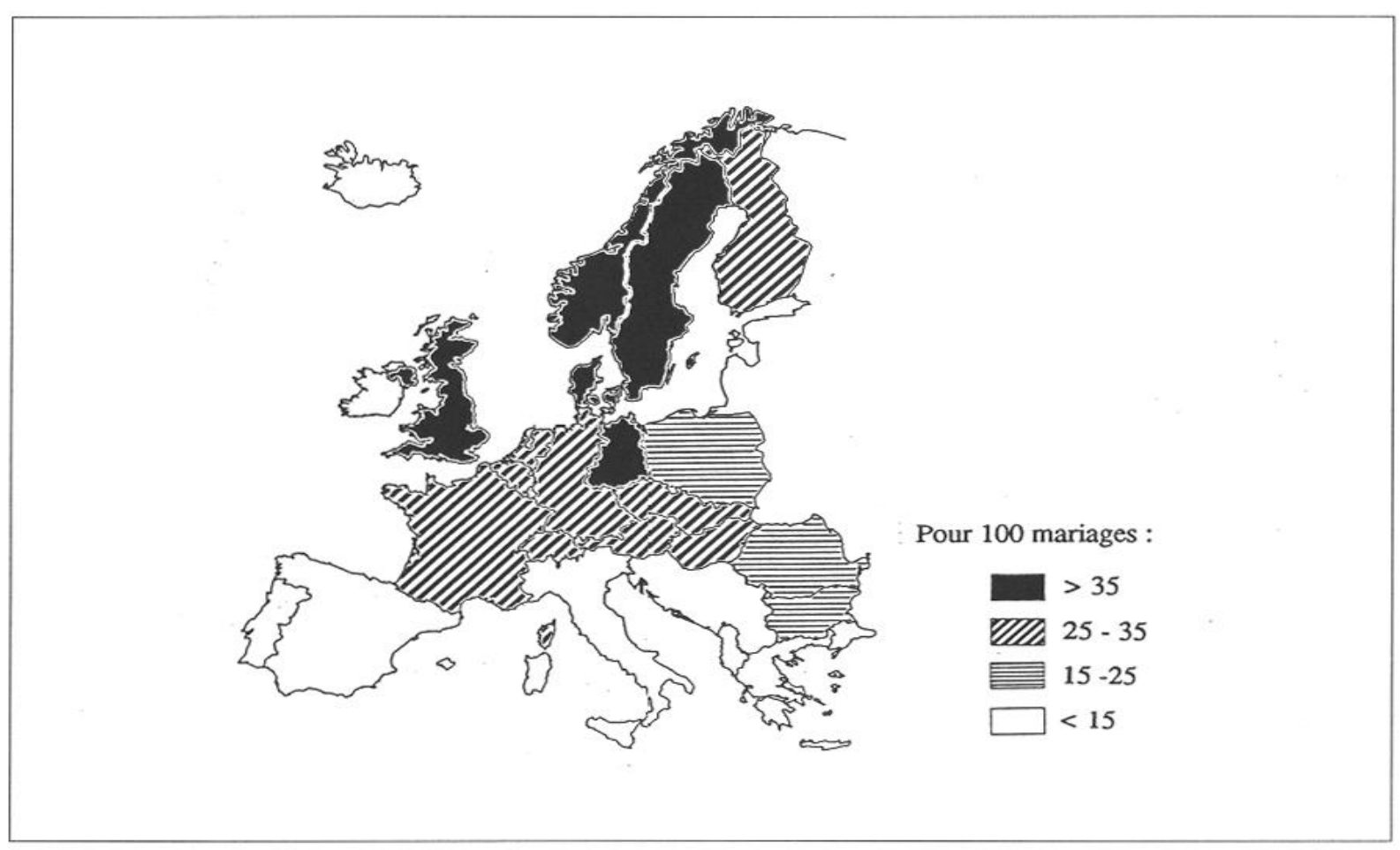

Carte 7

Les naissances hors mariage en Europe en 1988

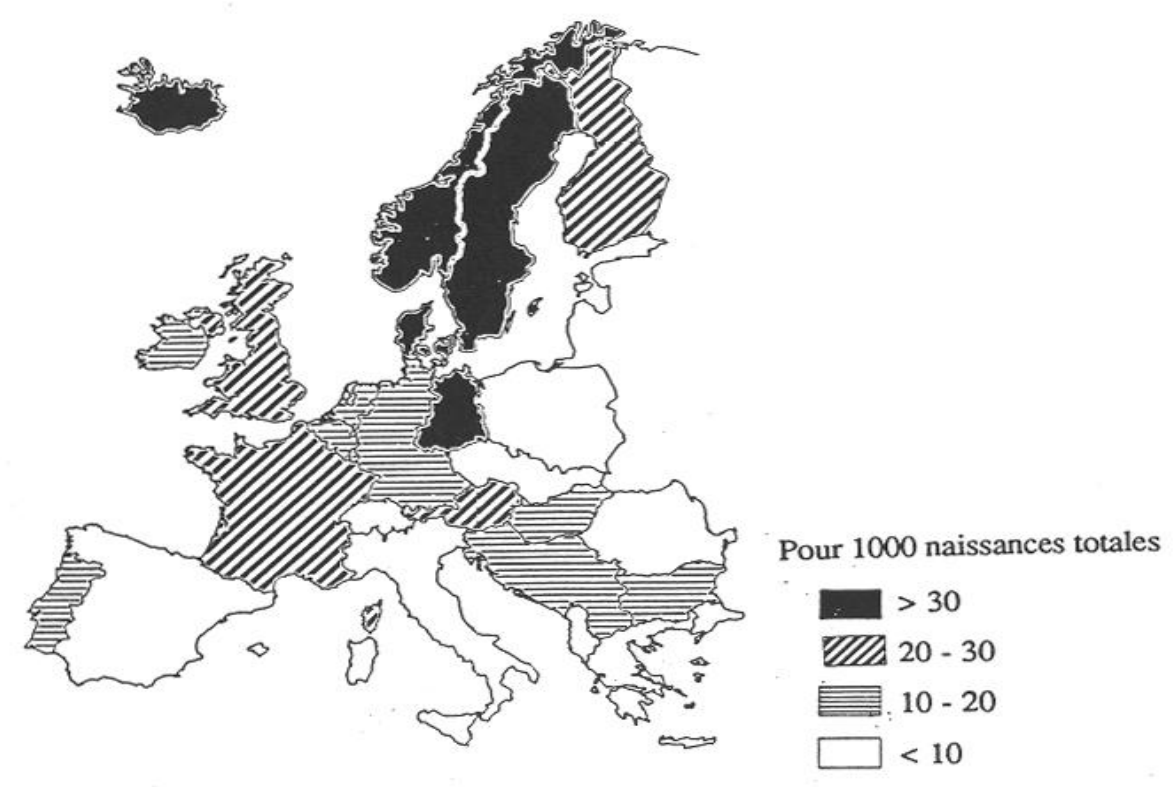


Souligner cette diversité n'est pas adhérer aux modèles culturalistes qui, de la coïncidence entre pratiques et aires culturelles, linguistiques ou religieuses, suggèrent l'existence d'un substrat imperméable au politique. Notre critique rejoint celle que le courant historiographique de la micro-analyse adresse aux modèles des sciences sociales qui postulent la stabilité des normes, qu'elles tireraient en particulier de leur ancienneté ${ }^{23}$. Les recherches menées dans le cadre de la micro-analyse montrent au contraire que, face aux normes et aux valeurs héritées, les attitudes sont toujours actives : le passé n'est pas appréhendé sous la forme de survivances mais comme un ensemble de ressources ; il n'est présent que parce qu'il est réactivé. Dans cette perspective, on pourrait lier les comportements démographiques des trois périodes politiques successives - l'avant-guerre, les quatre décennies socialistes et la période actuelle et ainsi éclairer les changements et les permanences.

Reste la question ultime des conséquences démographiques des changements politiques récents, ou autrement dit, la question de la prévision à laquelle la démographie prétend répondre. A rebours de cet usage ${ }^{24}$, on ne projettera pas les tendances, quelles soient récentes ou anciennes, quels que soient les paramètres socio-économiques pris en compte pour les infléchir. L'évolution de l'aprèsguerre, qui vient d'être décrite, comporte assez d'imprévisions pour en dissuader. On peut en revanche établir les conditions de changements démographiques - de la baisse de la surmortalité adulte, par exemple, ou bien de la chute du niveau de la fécondité - en réévaluant, sous les nouveaux régimes politiques, les facteurs institutionnels qui ont permis d'expliquer les divergences entre l'Est et l'Ouest. Par-delà leurs transformations ${ }^{25}$ (nouveau système de santé, disparition des législations familiales...), c'est le développement d'un nouveau type de rapport liant les individus aux institutions qui s'avérera déterminant. Cette nouvelle citoyenneté ne découle pas automatiquement de l'instauration de régimes démocratiques $^{26}$, elle fait encore défaut dans plusieurs anciens pays de l'Est. La diversité des expériences nous laisse, là aussi, avec de grandes incertitudes.

23. Voir sur ce sujet, Les formes de l'expérience, Bernard Lepetit (éd.), Albin Michel, 1985.

24. Tous les démographes ne se livrent pas à des projections, certains en critiquent même l'usage. Cf. Hervé Lebras, Les limites de la planète, Flammarion, 1994.

25. Il faut à la fois comprendre les ruptures institutionnelles, la volonté d'une distance avec le passé et le fait que les nouvelles formes se construisent avec des éléments hérités, transmis par le passé mais combinés selon des procédures nouvelles. Les travaux d'Alain Cottereau sur la période post-révolutionnaire française nous paraissent sur la question de la rupture politique particulièrement intéressants. Sur l'apport de ce travail, voir l'article de B. LEPETIT, «Le présent dans l'histoire », in Les formes de l'expérience, op. cit.

26. Voir en particulier l'article de Xavier GAULlIER, « Modernisation et État-providence en Europe centrale », Revue française des Affaires sociales, janvier-mars 1992. 